\title{
Multi-User Detection Performance Demonstrator for Realistic High Throughput Satellite Systems
}

\author{
Karin Plimon, Johannes Ebert, Nemanja Stamenic, \\ Harald Schlemmer \\ Institute for Information and Communication Technologies \\ JOANNEUM RESEARCH \\ Graz, Austria \\ Marius Caus \\ Communication Systems Division \\ Centre Tecnologic de Telecomunicacions de Catalunya \\ Castelldefels, Spain
}

\author{
Karin Plimon, Wilfried Gappmair \\ Institute of Communication Networks and Satellite Com- \\ munications \\ Graz University of Technology \\ Graz, Austria
}

\author{
Martina Angelone, Alberto Ginesi \\ ESTEC \\ European Space Agency \\ Noordwijk, The Netherlands
}

\begin{abstract}
Multi-beam satellite systems gain capacity from frequency reuse in the forward link, but they suffer from intersystem interference problems. Multi-user detection (MUD) is an advanced interference mitigation technique applied at the user terminal to cope with co-channel interferences. This paper complements theoretical studies on the efficiency of MUD by the development of a system performance demonstrator allowing the analysis of MUD performance limits under realistic channel conditions. A two-color system is assumed and compared to a traditional four-color application, which is to be used as a benchmark. By the application of MUD, a performance gain in peak and system throughput can be observed, while requiring higher complexity at the user terminal.
\end{abstract}

Keywords-Multi-user detection; Interference mitigation; Forward link; Frequency re-use; High throughput satellite system.

\section{INTRODUCTION}

Interference mitigation is the central paradigm in high throughput satellite systems (HTS) where multiple spot beams are operated with an aggressive frequency reuse factor [1]. An aggressive frequency reuse seeks to increase the system capacity but introduces at the same time intra-system interferences. With this technique, traditional noise-limited multi-beam satellite systems that are usually operated with a coloring scheme of 4 (FR-4) in the user downlink, transform to an interferencelimited system, operated with a frequency reuse 2 (FR-2) or even a full frequency re-use (FR-1). Amongst other interference sources, the co-channel interference contributes significantly to the intra-system interferences in this setup, as analyzed in [2], and is therefore in the focus of this work.

On a forward link, the introduced interferences can be either mitigated at the gateway or the user terminal. An advanced interference technique in this context is MUD that is applicable at the user terminal. The concept of MUD is based on the idea

This work was done in the context of a project funded by the European Space Agency (ESA) Contract No. 4000111016/14/NL/AD. to detect and decode two interfering spot beams at a single user terminal, commonly described as a multiple input single output (MISO) broadcast channel. In the case of MUD the main carrier and strongest interferer are jointly decoded while the remaining interferers are treated as noise. MUD operates in a way that it mutually extracts the information from both carriers. Thus it benefits from the strong interference by the strongest interfering spot beam operated at the same color.

The application of MUD as intra-system interference mitigation technique in the forward link has been studied extensively in theory by [3], [4], [5] and [6]. There, the obtained results agreed that a certain gain, especially at receiver positions close to the beam border (e.g. at the $-3 \mathrm{~dB}$ cross-over level), could be achieved by this technique.

This paper contributes to these former investigations and examines the capacity and throughput of MUD applied to a forward link in a satellite system considering also the effects of channel impairments and non-perfect parameter estimates in the user downlink and user terminal, respectively. A softwarebased system demonstrator for the physical layer was implemented to simulate a HTS system in the forward link with conditions close to reality. This simulation-based analysis aims to confirm the predicted performance gain by the application of MUD and investigates the performance degradation due to system impairments. To simulate under realistic satellite system conditions, DVB-S2 [7] and DVB-S2x [8] modulation and coding schemes (MODCODs) are used in a super-frame structure.

The structure of the paper is as follows: In the next section the system scenario is explained. A short introduction on the multi-user detector is provided in Section III. The information flow and reception modes are introduced in Section IV. Section $\mathrm{V}$ gives an overview on the system demonstrator and Section VI discusses the performance analysis considering scheduling and adaptive coding and modulation (ACM). Section VII concludes the work. 


\section{SYSTEM SCENARIO}

This performance analysis compares a FR-4 with a FR-2 multi-beam system, where the FR-4 performance is considered as a benchmark for the FR-2 results. For this purpose, the most representative carrier-to-interference ratios (C/I) were used for the considered coloring schemes. Since the user terminal position in a FR-2 scheme is relevant for the interference level, different user terminal positions, represented by six interference scenarios, were investigated as well. Scenarios were identified for the coloring schemes FR-4 and FR-2, and the selected $\mathrm{C} / \mathrm{I}$ distributions investigated are listed in TABLE I. where $\mathrm{I}_{1}$ denotes the strongest interferer. It can be observed that for FR2 the 5 strongest interferers are considered. At the detector only the main carrier and the strongest interferer $\left(\mathrm{I}_{1}\right)$ are detected. The remaining interferers $I_{2}$ to $I_{5}$ are thus treated as noise. The assumed available spectrum for a Ka-band user link is 500 $\mathrm{MHz}$ for each polarization. In FR-4 this is split to $250 \mathrm{MHz}$ per spot beam. A high throughput satellite operating a two-color scheme however, has $500 \mathrm{MHz}$ bandwidth available in a single spot beam. When assuming constant carrier power for both cases, FR-4 and FR-2, the SNR in FR-2 drops by $3 \mathrm{~dB}$ due to the larger bandwidth. TABLE I. lists the considered scenarios for the FR-4 and FR-2 schemes, where the total C/I for FR-4 results in $15 \mathrm{~dB}$.

Fig. 1 shows the achievable signal-to-noise plus interference ratio (SNIR) at a given signal-to-noise ratio (SNR) for these scenarios. The SNIR is shown from the main carrier's perspective (C) (solid curve) and the strongest interferer's perspective $\left(\mathrm{I}_{1}\right)$ (dashed curve), denoted as $\mathrm{I}_{1} \mathrm{NIR}$, considering the main carrier and the interferers $\mathrm{I}_{2}$ to $\mathrm{I}_{5}$ as interference (I). The upper graph compares the SNIR and $\mathrm{I}_{1} \mathrm{NIR}$ for the different interference scenarios from $\mathrm{C} / \mathrm{I}_{1}=0 \mathrm{~dB}$ to $10 \mathrm{~dB}$, as in TABLE I. before MUD is applied. The lower graph shows the SNIR and $\mathrm{I}_{1} \mathrm{NIR}$ after the strongest interferer, i.e. either $\mathrm{C}$ or $\mathrm{I}_{1}$, depending on the perspective, is ideally removed.

Inspecting the results, it is evident that the FR-2 system is interference-limited, since even at $30 \mathrm{~dB}$ SNR the SNIR does not exceed $10 \mathrm{~dB}$.

TABLE I. CARRIER TO INTERFERENCE RATIO SCENARIOS

\begin{tabular}{|c|c|c|c|c|}
\hline \multicolumn{5}{|c|}{ FR-4 } \\
\hline $\mathbf{C} / \mathbf{I}_{\mathbf{1}} \mathbf{( d B )}$ & $\mathbf{C} / \mathbf{I}_{\mathbf{2}} \mathbf{( d B )}$ & $\mathbf{C} / \mathbf{I}_{\mathbf{3}}(\mathbf{d B})$ & $\left.\mathbf{C} / \mathbf{I}_{\mathbf{4}} \mathbf{( d B}\right)$ & $\left.\mathbf{C} / \mathbf{I}_{\mathbf{5}} \mathbf{( d B}\right)$ \\
\hline 22 & 22 & 22 & 22 & 22 \\
\hline
\end{tabular}

\begin{tabular}{|c|c|c|c|c|c|}
\hline \multicolumn{7}{|c|}{ FR-2 } \\
\hline ID & $\left.\mathbf{C} / \mathbf{I}_{\mathbf{1}} \mathbf{( d B}\right)$ & $\left.\mathbf{C} / \mathbf{I}_{\mathbf{2}} \mathbf{( d B}\right)$ & $\left.\mathbf{C} / \mathbf{I}_{\mathbf{3}} \mathbf{( d B}\right)$ & $\left.\mathbf{C} / \mathbf{I}_{\mathbf{4}} \mathbf{( d B}\right)$ & $\left.\mathbf{C} / \mathbf{I}_{\mathbf{5}} \mathbf{( d B}\right)$ \\
\hline $\mathrm{A}$ & 0 & 25 & 25 & 27 & 30 \\
\hline $\mathrm{B}$ & 2 & 26 & 26 & 27 & 30 \\
\hline $\mathrm{C}$ & 4 & 27 & 26 & 27 & 30 \\
\hline $\mathrm{D}$ & 6 & 27 & 26 & 27 & 30 \\
\hline $\mathrm{E}$ & 8 & 26 & 26 & 27 & 30 \\
\hline $\mathrm{F}$ & 10 & 22 & 26 & 27 & 30 \\
\hline
\end{tabular}
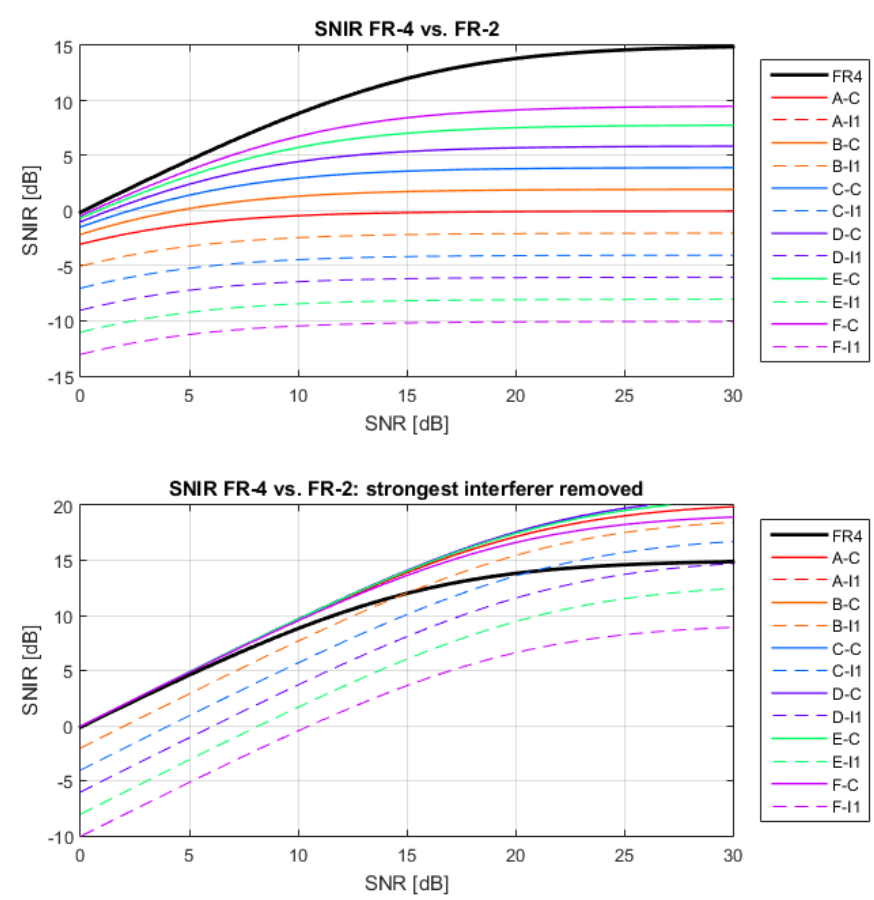

Fig. 1. SNIR comparison of FR-4 and FR-2, main carrier $\mathrm{C}$ and interferer $\mathrm{I}_{1}$

Once the strongest interferer, i.e. $\mathrm{C}$ or $\mathrm{I}_{1}$, can be fully detected and removed, the SNIR of the carrier of interest increases instantly since the remaining interferers (I) are quite low. The application of MUD is limited to FR-2 scenarios, since FR-1 would add too much complexity to the system [3] which leads to a potentially unstable behavior. For this reason FR-1 was excluded from further analysis.

\section{MULTI-USER DETECTOR}

The implementation of the multi-user detector follows the idea introduced by Studer et al. [9], [10]. The main concept of MUD is based on the knowledge of the transmitted MODCOD pair, provided via the start of the super-frame (SOSF) field, the channel estimation and soft bit detection. The iterative MUD concept as explained by Caus et al. in [6] is applied using joint detection of both carriers and iterative decoding. The decoded information of one carrier is provided as log-likelihood ratio (LLR) a-priori information for the decoding of the other. This concept is illustrated in Fig. 2. This joint detection process requires a structure that provides frames of constant symbol length regardless their modulation schemes. This has been foreseen already in the DVB-S2X [8] super-frame structure in the form of so-called bundled frames. This implementation of MUD assumes a frame-synchronous transmission, which can be practically achieved since the signals are transmitted by the same satellite.

A single MUD iteration invokes the detector and the decoder twice, once for each carrier. The overall performance therefore depends on the selected number of MUD as well as low-density parity check (LDPC) decoder iterations. The interleaver, shown in Fig. 2, is negligible for the processing speed. 


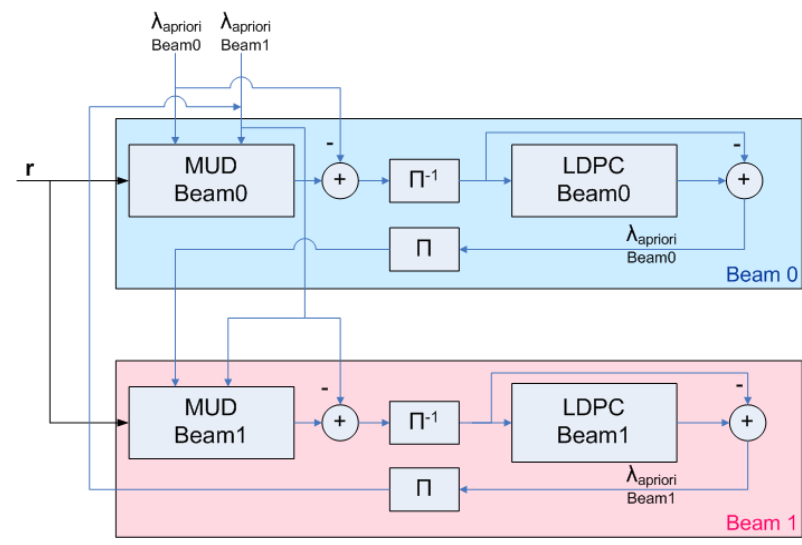

Fig. 2. Joint detection MUD structure based on [6]

\section{INFORMATION FLOW AND RECEPTION MODES}

MUD allows two possible flows of information. In the socalled single carrier (SC) mode the receiver uses the information transmitted by its own beam, whereas in MAC mode the user terminal takes the information from its dedicated carrier and the strongest interferer, but just for a fraction of time. In addition to MUD also the reception without MUD, i.e. interference treated as noise, is considered as well.

\section{A. Interference as Noise (IAN)}

Independent of the used coloring scheme, a user terminal has always the option to treat the present interferers as noise, shown in Fig. 3. There, the carriers of Beam0 $\left(\mathrm{B}_{0}\right)$ and Beam1 $\left(\mathrm{B}_{1}\right)$ are denoted as $\mathrm{C}^{\left(\mathrm{B}_{0}\right)}$ and $\mathrm{C}^{\left(\mathrm{B}_{1}\right)}$ and the applied MODCODs are represented by $m_{0}$ and $m_{1}$, respectively. This reception technique is typically applied in FR-4 systems. Theoretically it can be used also in FR-2 systems, but with an immensely degraded performance caused by the higher co-channel interference.

\section{B. MAC-Multi-user Detection (MAC-MUD)}

A user terminal in a FR-2 satellite system, equipped with MUD has the chance to receive information provided via two adjacent spot beams of the same color, demonstrated in Fig. 4. There, from the perspective of the user terminal in Beam0 (UT0) Beam1 is marked as the strongest interferer $\mathrm{I}_{1}{ }^{\left(\mathrm{B}_{1}\right)}$.

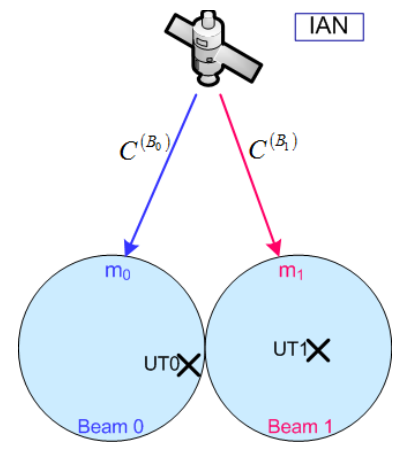

Fig. 3. FR-4 and FR-2 interference as noise (IAN) concept

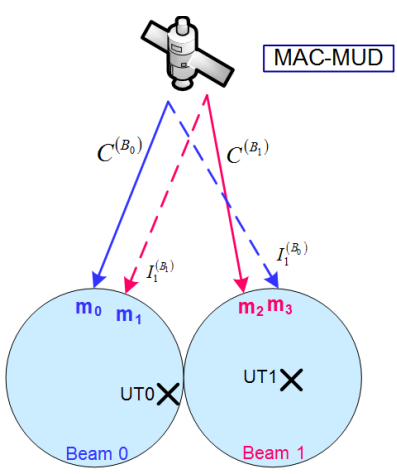

Fig. 4. FR-2 MAC-MUD concept

UT0 in this case receives information via two carries with its specific MODCOD pair $\left(\mathrm{m}_{0}, \mathrm{~m}_{1}\right)$. In order to balance the service, this is done in an alternating way with another user (e.g. UT1 operating the MODCOD pair $\left(\mathrm{m}_{2}, \mathrm{~m}_{3}\right)$ ) to serve them equally while increasing the capacity. The selection of the MODCODs that are assigned to both beams can be done by the scheduler based on the individual conditions for the single user terminal. An ideal selection thus nominates the MODCOD pair with highest spectral efficiency with regard to the local interference conditions at the terminal.

\section{Single Carrier - Multi-user Detection (SC-MUD)}

Despite the fact that a user terminal is operated in a FR-2 setup, the terminal can still be served only by a single spot beam. In this case the terminal has the option, in contrast to the situation discussed in Subsection A. before, to use the interfering beam to decode the own carrier using MUD and discard the interferer information, called SC-MUD. This approach is shown in Fig. 5. Assuming this case, the scheduler has to optimize the efficiency taking into account the interference conditions at both user terminal positions since the MODCOD pairs are not independent. Optimizing only for a single terminal would penalize the second user terminal since the MODCOD pair in this case is not specifically dedicated to a single user terminal. This would result in an imbalance in the network. While the information provided via the interferer is irrelevant for the user terminal, the MODCOD used by the interferer (i.e. $\mathrm{m}_{1}$ for the perspective of UT0 and $\mathrm{m}_{0}$ for the perspective of UT1) is still of utmost importance for the MUD process.

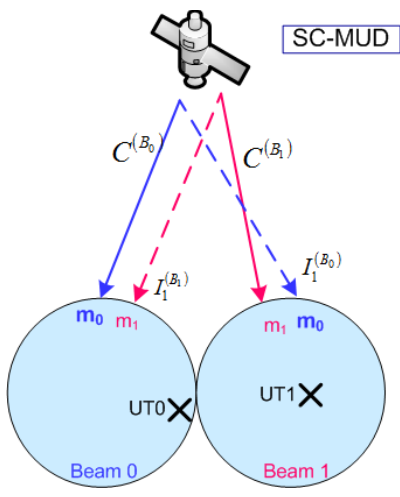

Fig. 5. FR-2 SC-MUD concept 


\section{SYSTEM DEMONSTRATOR AND COMMUNICATION LINK}

The system demonstrator is a module-based software tool implemented in $\mathrm{C}++$ and versatile for a broad spectrum of satellite communication applications on the physical layer. The configuration of a simulation is described by a simulation file specifying the order and the input parameters of the applied modules. Performance simulations up to FER $=1$ E- 6 were required for this performance comparison, which means that the program execution time was crucial. A sufficient execution speed was achieved by a computation on a 96 core grid realized by 3 rack units.

The system demonstrator represents the following link components, categorized in transmitter, channel and receiver part, as shown in Fig. 6. The aim was to simulate a physical layer satellite link that is close to reality. Therefore, transponder impairments [8], i.e. HPA nonlinearities, phase noise, IMUX/OMUX filters [13], as well as parameter estimation and carrier synchronization [11], [12] were considered in addition to the AWGN channel. A total of 6 beams were considered, where the main carrier and the strongest interferer $\left(\mathrm{I}_{1}\right)$ are configurable in terms of MODCOD and frame length. The remaining interferers $\left(\mathrm{I}_{2}-\mathrm{I}_{5}\right)$ however, are treated as noise.

The analysis of theoretic system performances assuming ideal synchronization and no impairments results only in rough approximation results since significant impairments in the order of several $\mathrm{dB}$ can be introduced depending on the selected modulation. The capability of MUD leads to a complex behavior that is aggravated by the effects of phase noise, nonlinearities, synchronization and parameter estimation. The carrier identification is done by Walsh-Hadamard (WH) codes. The estimation of the symbol timing, carrier frequency/phase and signal amplitude is achieved by the SOSF field.

\section{PERFORMANCE ANALYSis}

The selected parameter setting allowing a performance analysis in a realistic environment is shown in TABLE II. The timing and frequency error at the receiver refer to cold acquisition. The maximum number of iterations for the detector and decoder were selected based on performance analysis and the early stop criterion was activated. The MUD performance of DVB-S2 and DVB-S2X MODCOD pairs, considering the modulations QPSK, 8PSK, 16APSK and 32APSK was assessed in terms of spectral efficiency and throughput. Based on these results, lookup tables for each FR-2 scenario were generated taking the envelope MODCOD pairs. In the course of the analysis it revealed that 32APSK is not feasible under these impaired conditions which means that other techniques, such as pre-distortion to counteract the nonlinearities at the HPA, would be required.

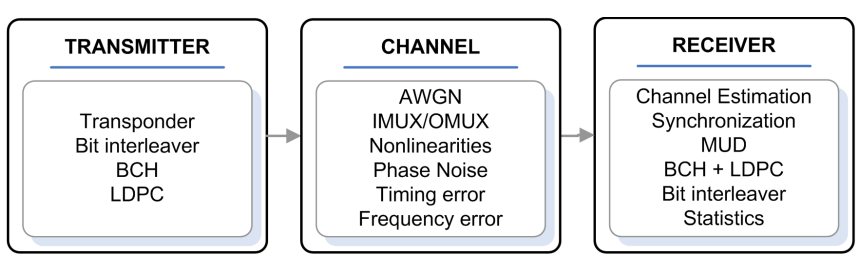

Fig. 6. Schematic link concept of the system demonstrator
TABLE II. PARAMETER SETTING

\begin{tabular}{|l|c|}
\hline \multicolumn{1}{|c|}{ Performance } & (unit) \\
\hline Timing error $@$ RX & $100 \mathrm{ppm}$ \\
\hline Frequency error $@$ RX & 0.001 \\
\hline Phase noise mask & VSAT_P2 [8] \\
\hline OBO & $1.7 \mathrm{~dB}$ \\
\hline LDPC & offset min-sum \\
\hline LDPC max iterations & 200 \\
\hline MUD max iterations & 4 \\
\hline Early stop & active \\
\hline
\end{tabular}

Due to this finding, 32APSK was excluded from the analysis presented in the sequel. The total degradation of 16APSK became therefore the critical factor for the selection of the OBO. For fair comparison between FR-4 and FR-2 the results in the following are compared with respect to the power in the beam center at FR-4, implying that $+3 \mathrm{~dB}$ noise was considered for FR-2 due to the higher bandwidth of $500 \mathrm{MHz}$.

\section{A. MUD Performance}

The spectral analysis of MODCOD pairs for all FR-2 scenarios was carried out twice. In the first step the analysis was done only for MUD and then for the setup considering the specific set of system impairments, as introduced before, with non-perfect parameter estimates in order to understand the degree of degradation. Analyzing the envelope of the achieved spectral efficiencies revealed that the results of both iterations were composed of different MODCOD pairs, confirming a sensitivity related to the selected MODCOD pair. In Fig. 7 a sample envelope of MUD spectral efficiencies is demonstrated for the scenario $\mathrm{FR}-2, \mathrm{C} / \mathrm{I}_{1}=6 \mathrm{~dB}$. This figure compares the MAC-MUD performance of AWGN only with the impaired system. The obtained results show a gap in the higher SNR region for the impaired case. This gap can be explained by the fact that low-order modulations are used for MUD in this case also in higher SNR regions that are at the same time less affected by the impairments and thus keep a stable throughout.

Looking at the instantaneously achievable peak throughput in Fig. 8, it is remarkable that MAC-MUD practically doubles the peak throughput of a classical FR-4.

Confirming this fact is especially beneficial for the satellite operator since it provides much flexibility to use and share the capacity amongst the users and thus meet the individual traffic requirements as predicted in [4]. The FR-4 performance in this context is only understood as reference performance for comparison purposes. 


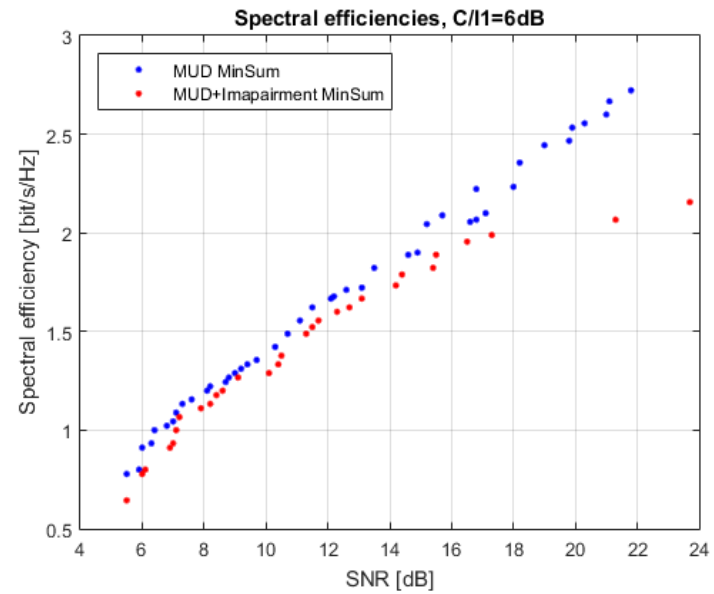

Fig. 7. FR-2 MUD spectral efficiency envelope comparison with impairment

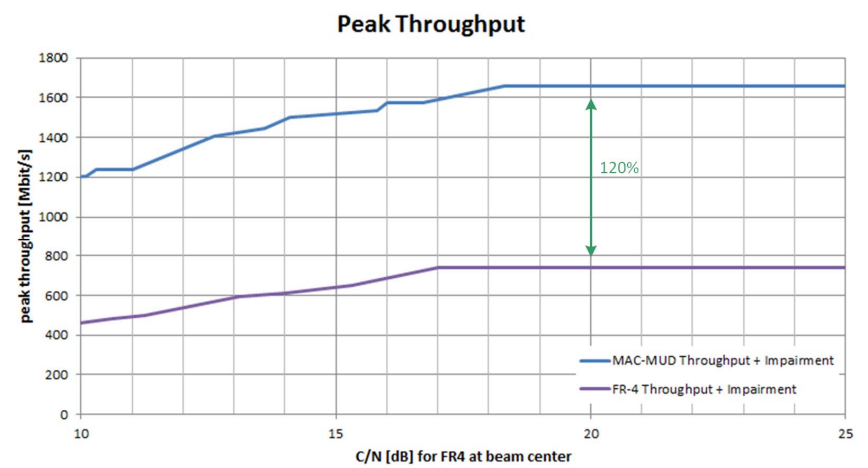

Fig. 8. Instantaneous peak throughput comparison FR-2 with FR-4

\section{B. Scheduling}

The scheduler selects the best suitable technique depending on the current situation for the user on ground. Scheduling algorithms for MUD were tested to determine whether a combination of a low-high $\mathrm{C} / \mathrm{I}_{1}$ scenario in the two beams or an equal setting for both user terminals optimizes the throughput performance. It revealed that dissimilar C/I pairs achieve performance results comparable to a random pairing. The scheduler therefore draws for each beam one of the considered C/I distributions, resulting in uniformly distributed user terminal positions. The bandwidth dissimilarity is also the reason why FR-4 has a significantly better performance w.r.t. spectral efficiency but is excelled by FR-2 in terms of throughput. More explicitly this means that for FR-2 the scheduler selects IAN, MACMUD or SC-MUD resulting in a performance envelope that marks the best achievable result.

\section{Adaptive Coding and Modulation}

In the last step, the tropospheric effects were considered for the link as well, where the rain attenuation, representing the strongest impact of the troposphere, was added as impairment to the system. For this purpose the rain attenuation CCDF from the area of Graz/Austria was used as example. With this effect the adaptive coding and modulation (ACM) system perfor- mance in terms of availability can be assessed as well. In Fig. 9 the method-specific spectral efficiency performance is shown. A slight gain of FR-2 SC-MUD compared to FR2-IAN can be observed caused by the application of MUD at the receiver. Inspecting the performance at FR-2 reveals that SC-MUD performs better at lower SNR ranges and MAC-MUD is applicable at $\mathrm{C} / \mathrm{N}$ values beyond $15 \mathrm{~dB}$. By the application of the scheduler the envelope performance for FR-2 can be obtained.

Comparing the average spectral efficiency envelopes of FR-4 and FR-2 in Fig. 10 illustrates that the spectral efficiency is less meaningful as performance measure due to the bandwidth discrepancy between FR-4 and FR-2.

For this reason special emphasis is put on the average system throughput as performance measure, shown in Fig. 11. Considering a $\mathrm{C} / \mathrm{N}$ in the range of $20 \mathrm{~dB}$, a gain of approx. $15 \%$ can be observed compared to a traditional FR-4 setting while keeping an availability of $99.98 \%$.

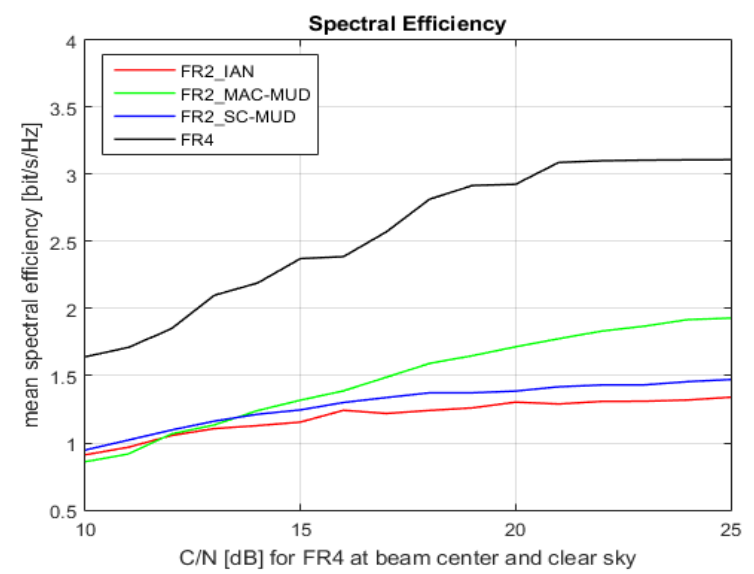

Fig. 9. Individual spectral efficiency performances for FR-2 and FR-4

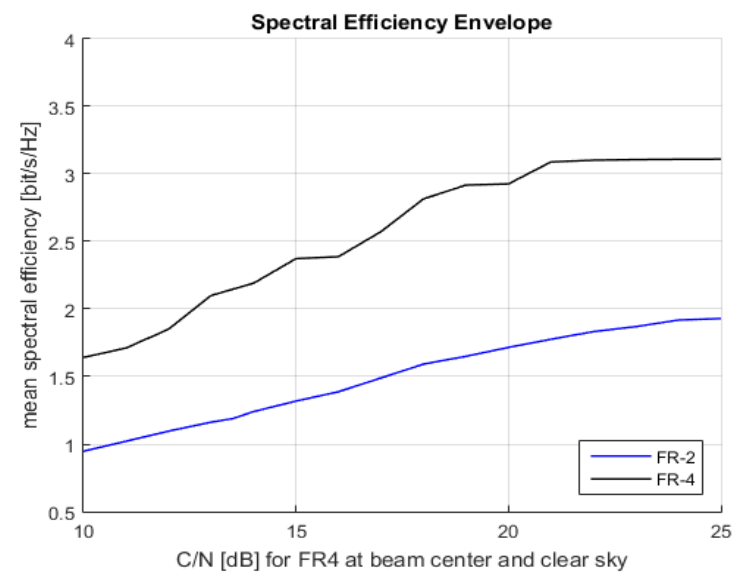

Fig. 10. Average spectral efficiency with impairment and ACM 


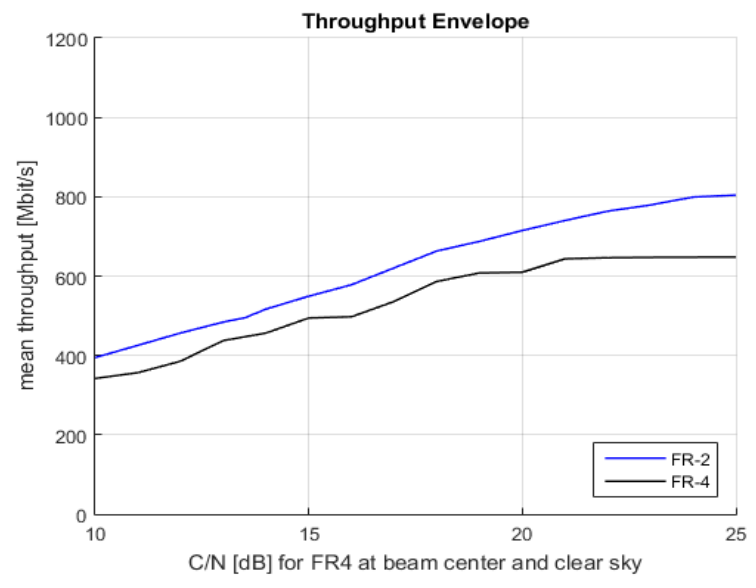

Fig. 11. Average system throughput with impairment and ACM

\section{CONCLUSIONS}

This work investigated the achievable performance gain in a HTS system close to reality by the application of MUD. A two coloring frequency reuse was considered for this setup. Performance tables were generated based on physical layer simulations to identify the most efficient MAC-MUD MODCOD pairs for the defined interference scenarios. These results were used to determine the instantaneous peak throughput as well as the average system performance in terms of spectral efficiency, throughput and availability using an impaired forward link. System impairments such as nonlinearities, phase noise and non-perfect parameter estimates were considered for this purpose.

Three different FR-2 transmission modes were examined and compared to FR-4 as benchmark. A gain in terms of peak and system throughput, as predicted by [4], was confirmed for MAC-MUD in presence of system impairments and ACM.

The obtained results show that MUD achieves a clear performance gain around $\mathrm{C} / \mathrm{N}=20 \mathrm{~dB}$ and higher which is in practice not easily achievable with HTS link budgets. The present gain in terms of peak throughput though demonstrates the resource flexibility of this detection method. Nevertheless, despite the observed gain, the required complexity at the receiver must not be neglected.

\section{ACKNOWLEDGMENT}

The authors would like to thank Eral Tuerkyilmaz for his dedicated contribution to this work.

\section{REFERENCES}

[1] H. Fenech, S. Amos, A. Tomatis, and V. Soumpholphkakdy, "KA-Sat and future HTS systems," in Proc. IEEE International Vacuum Electronics Conference, Paris, France, pp. 1-2, May 2013.

[2] E. Lutz, "Co-channel interference in high-throughput multi-beam satellite systems," in Proc. IEEE Satellite and Space Communications, London, UK, pp. 885-891, June 2015.

[3] S. Andrenacci et al., "Physical layer performance for multi-user detection in broadband multi-beam systems based on DVB-S2," in Proc. European Wireless, Barcelona, Spain, pp. 561-565, May 2014.

[4] M. Angelone, A. Ginesi, M. Caus, A. I. Perez-Neira, and J. Ebert, "System performance of an advanced multi-user technique for high throughput satellite systems," in Proc. 21st Ka and Broadband Communications Conference, Bologna, Italy, pp. 1-9, October 2015.

[5] G. Colavolpe, A. Modenini, A. Piemontese, and A. Ugolini, "Multiuser detection in multibeam satellite systems: theoretical analysis and practical schemes," IEEE Transactions on Communications, vol. 65, issue 2, pp. 945-955, February 2017.

[6] M. Caus, A. I. Perez-Neira, M. Angelone, and A. Ginesi, "An innovative interference mitigation approach for high throughput satellite systems," in Proc. International Workshop on Signal Processing Advances in Wireless Communications, Stockholm, Sweden, pp. 515-519, June 2015.

[7] Digital video broadcasting (DVB); Second generation framing structure, channel coding and modulation systems for broadcasting, interactive services, news gathering and other broadband satellite applications, EN 302 307, ETSI Standard, 2004.

[8] Digital video broadcasting (DVB); Second generation framing structure, channel coding and modulation systems for broadcasting, interactive services, news gathering and other broadband satellite applications; Part II: S2 Extensions (S2-X). ETSI Standard, 2015.

[9] C. Studer, A. Burg, and H. Bölcskei, "Soft-output sphere decoding: algorithms and VLSI implementation," IEEE Journal on Selected Areas in Communications, vol. 26, pp. 290-300, February 2008.

[10] C. Studer and H. Bölcskei, "Soft-input soft-output single tree-search sphere decoding," IEEE Ttransactions on Information Theory, vol. 56, pp. 4827-4842, October 2010.

[11] W. Gappmair and A. Ginesi, "Cramer-Rao Lower Bound and Parameter Estimation for Multibeam Satellite Links," Int. Journal Satellite Commun. Network., vol. 35, pp. 343-357, 2017.

[12] W. Gappmair, K. Plimon, and A. Ginesi, "Coarse Recovery of Carrier Frequency and Symbol Timing in Multibeam Satellite Channels," Int. Journal Satellite Commun. Network., available online, 2018.

[13] A. Ginesi et al., "DVB-S2X channel models," TM-S2 Channel Model Group, May 2014. 